\title{
La langue corse dans l'enseignement : données objectives et sens sociétal
}

\section{Alain Di-Meglio}

\section{(2) OpenEdition \\ Journals}

Édition électronique

URL : http://journals.openedition.org/trema/975

DOI : 10.4000/trema.975

ISSN : 2107-0997

Éditeur

Faculté d'Éducation de l'université de Montpellier

Édition imprimée

Date de publication : 1 septembre 2009

Pagination : 85-94

ISSN : 1167-315X

Référence électronique

Alain Di-Meglio, «La langue corse dans l'enseignement : données objectives et sens sociétal », Tréma [En ligne], 31 | 2009, mis en ligne le 01 septembre 2011, consulté le 19 avril 2019. URL : http:// journals.openedition.org/trema/975; DOI : 10.4000/trema.975

Ce document a été généré automatiquement le 19 avril 2019

Trema 


\title{
La langue corse dans l'enseignement: données objectives et sens sociétal
}

\author{
Alain Di-Meglio
}

1 L'état chiffré de l'enseignement du corse est l'objet de nombreuses enquêtes nées du besoin de planification de différentes institutions ou collectivités territoriales en Corse. Ainsi l'Académie de Corse rend publiques un certain nombre de données statistiques. D'autres sources comme l'INSEE Corse ou des enquêtes de l'Université dans le cadre de recherches peuvent encore contribuer à une meilleure idée de l'état de l'enseignement d'une jeune ${ }^{1}$ discipline: la Langue et Culture Corses (désormais LCC).

2 Ces sources se recoupent ou se retrouvent souvent dans une littérature de rapports ou d'articles de presse soit pour bâtir un diagnostic, soit dans un simple but

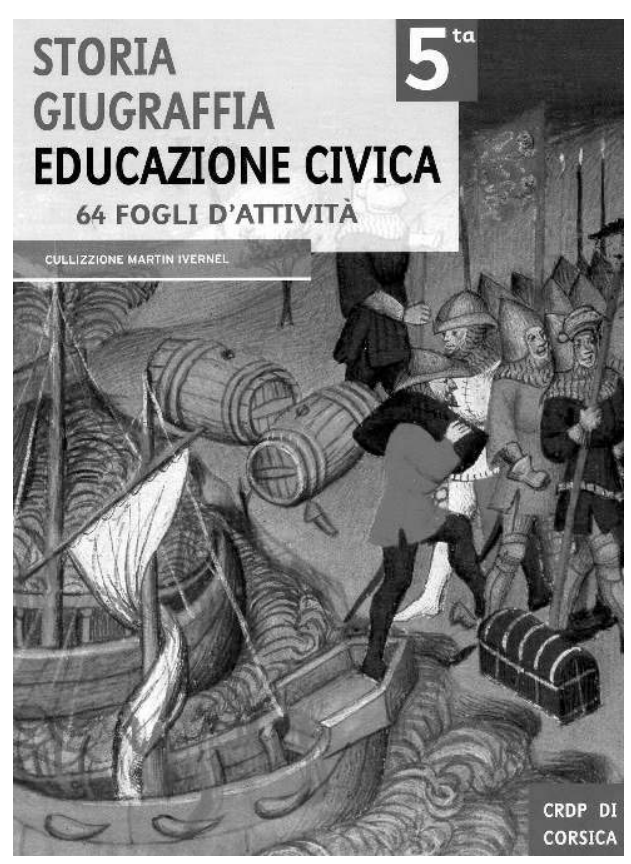
d'information. Car la langue corse dans la société est devenue tout à la fois un vrai consensus politique et, sur des aspects comme la co - officialité des langues corse et française ou la généralisation obligatoire de l'enseignement de la langue régionale, un enjeu encore marqué pour l'avenir de l'île. Les deux considérations se rejoignent dans le fait que les aspects diagnostiques tant dans la présentation que dans les représentations revêtent une grande importance. 
La question de la langue corse est donc socialement vive et intègre régulièrement le débat sur l'avenir politique de l'île. Cela se marque, entre autres, par le basculement total de cet enseignement dans le service public.

On constate aujourd'hui la disparition de l'enseignement associatif qui pour avoir été pionnier à la fin des années 1960 a cédé le pas à l'éducation publique en Corse à mesure que celle - ci renforçait son cadre statutaire et que la régionalisation augmentait ses aides à des fins d'accompagnement. Le secteur privé n'a pour sa part jamais été très important en Corse ${ }^{2}$.

On constate par ailleurs une augmentation constante des effectifs avec une très forte croissance à partir des années 1980 par les effets cumulés de la décentralisation et de l'évolution statutaire de l'enseignement et des enseignants (création de la licence LCC puis du CAPES).

\section{Effectifs et statuts : des progrès importants}

Il est vrai que si l'on compare l'état statutaire du corse enseigné au début des années 70 à celui d'aujourd'hui, la différence est de taille. Elle souligne plus de trente ans d'évolutions soumises aux soubresauts de la vie politique dans cette période.

7 Globalement la langue corse a bénéficié normalement des mesures en faveur des langues régionales en $\mathrm{France}^{3}$. $\mathrm{Au}$ - delà, elle jouit d'une double spécificité : les prérogatives de la Collectivité Territoriale de Corse (désormais CTC) de 1992 qui prévoit dans son article 53 que l'Assemblée « adopte et met en œuvre un plan de développement de l'enseignement de la langue et de la culture corses, et de promotion de la langue dans la société » et la loi du 22 janvier 2002 relative à la Corse, et de l'article 7 qui prévoit que le corse est une matière «enseignée dans le cadre de l'horaire normal des écoles maternelles et élémentaires ».

Les effets les plus importants se ressentent surtout au niveau des cadres statutaires :

- pour l'enseignement supérieur, la 73e section des universités compte à l'Université de Corse 5 professeurs, 7 maîtres de conférences et 10 certifiés (PrCe) ; ils donnent des enseignements dans la filière d'études corses jusqu'au master et dans l'ensemble des autres filières pour l'obtention d'un certificat conforme aux normes européennes (CLES);

- pour l'enseignement secondaire, le concours du CAPES a donné en 18 ans d'existence plus de 120 professeurs certifiés dont 113 affectés dans les classes du secondaire (soit dans les 97 postes de l'Académie, soit en brigade de remplacements);

- l'enseignement en maternelle et en élémentaire bénéficie aujourd'hui de $25 \%$ des maîtres titulaires d'une habilitation à l'enseignement bilingue. Ils sont 305 selon les chiffres de l'IA - IPR - LCC ${ }^{4}$ dont 104 recrutés directement par le concours spécifique (cf. note 2). On sait par ailleurs, selon l'enquête de l'IGEN $(2006,87)$, qu'environ $40 \%$ des postes du primaire sont réservés au concours spécifique.

Les professeurs titulaires des premier et second degrés évoluent dans l'ensemble des établissements scolaires. Toutefois, l'accent est à mettre sur l'évolution des sites bilingues (proportions dans le graphique ci - dessous) qui répondent à une logique de bassin géographique, de manière à assurer un enseignement bilingue filé sur l'ensemble du cursus scolaire (de la maternelle au bac). 


\section{Un élève de LCC sur huit en classe bilingue \\ Répartition des elèves suivant un enseignement de langue et culture corses (LCC) en 2004/2005}

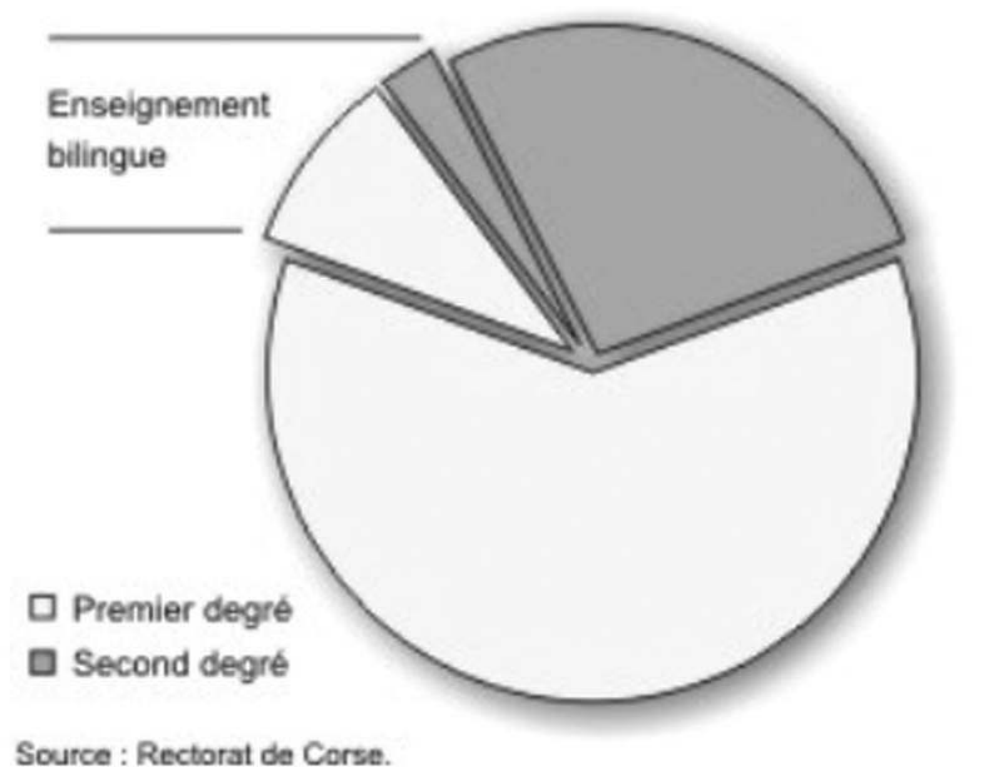

L'IGEN $(2006,87)$ souligne aussi le développement de l'enseignement bilingue comme le fait le plus marquant: "L'enseignement bilingue est en forte progression. Il est actuellement assuré sur 33 sites avec les principes suivants :

- un cursus bilingue doit être ouvert dans toute école maternelle à au moins quatre classes et un choix d'implantation de sites d'écoles élémentaire doit être réalisé par secteur de collège pour préparer l'accueil en 6e d'élèves susceptibles de suivre les cours en filière bilingue : le nombre de professeurs habilités augmente rapidement ; la visite de sites bilingues par la mission atteste de la progression de cet enseignement ;

- une poursuite d'études est garantie dans le second degré, [...];

- des sections bilingues à dominante « langues romanes » doivent conduire à des sections européennes ou méditerranéennes en lycée. » 


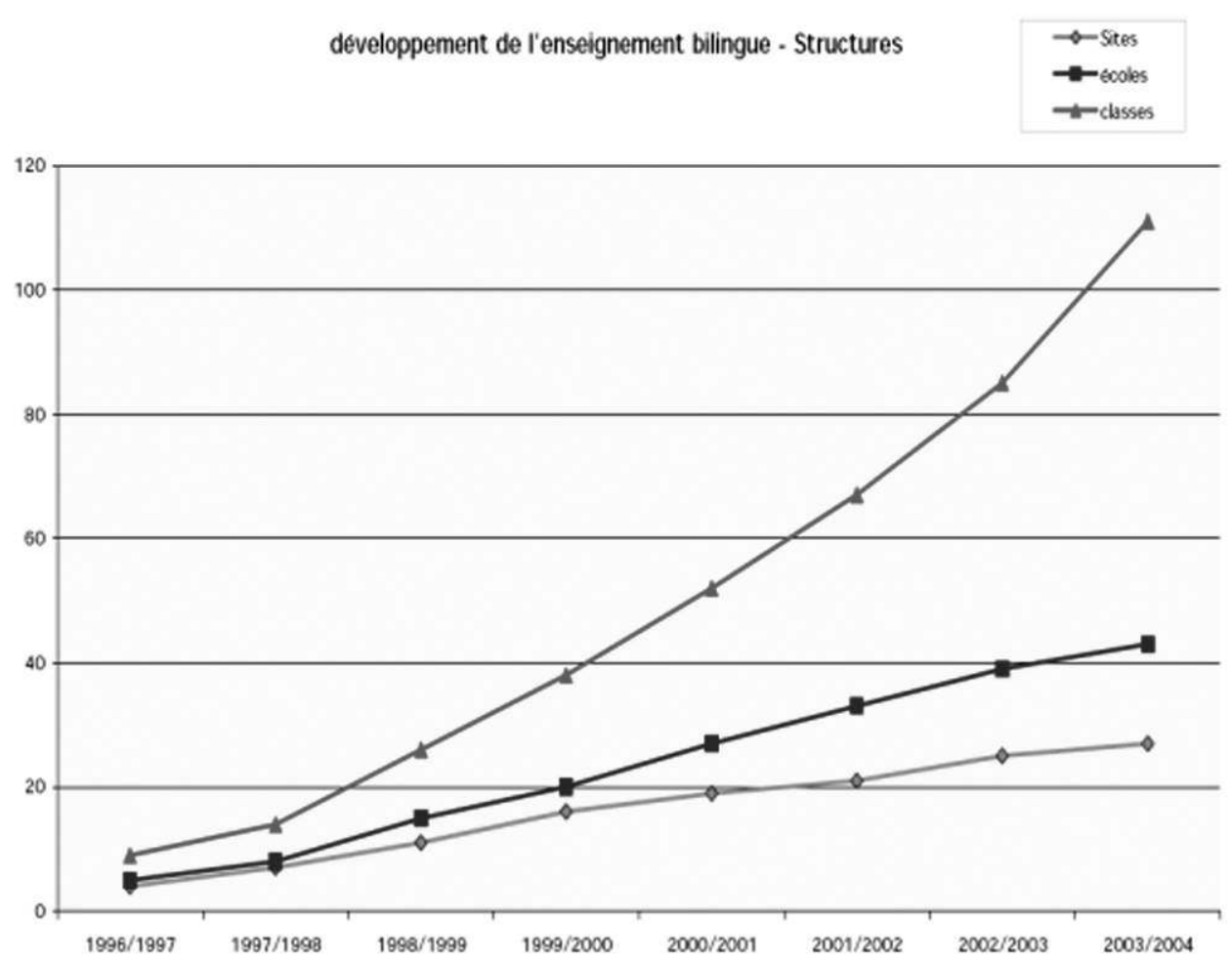

On notera par ailleurs une remarque sur le deuxième point concernant la poursuite des études sur un cursus régulier : le rapport signale « le risque de constituer des classes à la composition figée depuis l'école maternelle, voire celui de classes regroupant uniquement des enfants de parents corses. Cela nécessite par ailleurs le fléchage de postes bilingues (mathématiques et corse, histoire et géographie et corse, documentaliste et corse) dans les collèges, ce qui laisse parfois des emplois vacants par manque de candidats habilités, ou crée des rigidités nouvelles dans les répartitions de service ».

Nous touchons ici l'un des aspects polémiques du développement de l'enseignement bilingue. Le fléchage des postes pose incontestablement un problème de différence de statut des maîtres (bilingues habilités et standards) dans le mouvement des postes.

En même temps, les militants du bilinguisme dénoncent depuis longtemps le caractère haché, presque systématiquement incomplet du cursus bilingue. Ajoutons à cela des effets pervers bien connus des sociologues de l'éducation et que Sébastien QUENOT $(2009,75)$ présente ainsi : « Le caractère facultatif de l'enseignement conduit dans chaque situation à des stratégies d'évitement contraires à la mixité sociale, et à l'équité. Compte tenu de la ségrégation scolaire constatée dans certains sites, des stratégies d'évitement d'enfants ou d'enseignants mises en œuvre et de la portée sociétale attribuée à l'enseignement bilingue, la question du libre choix répond certes aux droits auxquels aspirent les consommateurs - citoyens des démocraties libérales mais il interroge notre conception de l'équité et du vivre ensemble. »

On constate donc un certain nombre de problèmes liés au développement et à la massification des effectifs des maîtres et des élèves en filière bilingue mettant dans une concurrence imprévue la légitimité d'un enseignement efficace de la langue corse dans un cadre reconnu et les valeurs du commun offert à tous. Cette considération aboutit dans une frange de l'opinion à la revendication d'un enseignement bilingue généralisé et obligatoire. 


\section{Les modalités d'enseignement}

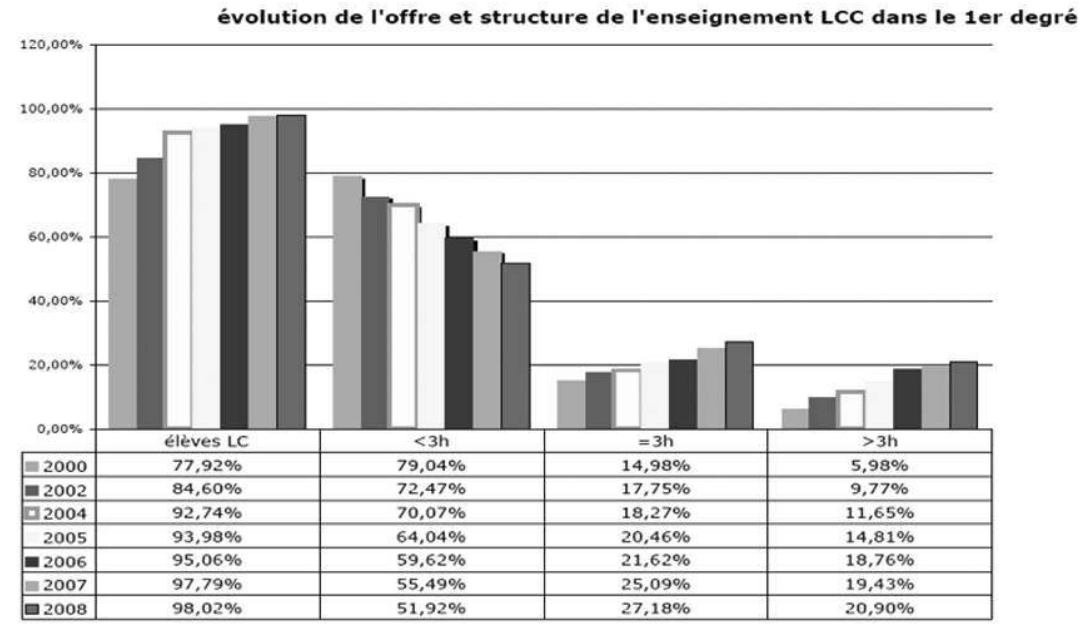

Les évolutions des effectifs premier degré ci - dessus (source Rectorat de Corse) montrent bien les catégorisations issues des différents statuts au cours de ces trente dernières années.

Le premier degré affiche à ce titre une situation globale très disparate. Ici le tableau situe l'ensemble des données autour d'une sorte de norme issue de la circulaire SAVARY de 1982 qui fixe l'enseignement à 3 heures / semaine. L'avènement des filières bilingues permettra de conjuguer un enseignement en immersion partielle et 3 heures d'études de la langue dont le cumul avoisinerait $50 \%$ de l'enseignement dispensé. Ce cas idéal ne constitue pas la majorité y compris au sein même des classes ayant le statut bilingue.

Nous avons donc théoriquement deux grands cas de figure (primaire et secondaire confondus) dans un cadre global ainsi présenté par le rapport de l'IGEN $(2006,85)$ : « Par principe cet enseignement est « obligatoire » pour le service public et «facultatif » pour les familles » :

- Un enseignement de 3 heures ; il est offert de façon généralisée à l'école primaire. Il est alors assuré directement par le professeur des écoles qui peut le déléguer à un tiers, soit par décloisonnement avec un autre collègue, soit en le confiant à un intervenant extérieur. Pour le secondaire, il est offert en option facultative à partir de la 6e puis il entre dans les possibilités de LV2 ou LV3 à partir de la 4e. Les enseignements sont assurés par un professeur certifié LCC.)

- La possibilité de recevoir un enseignement bilingue donné par un professeur habilité. Cette habilitation pour le premier degré s'obtient directement par le concours spécifique ou par un examen organisé sous la présidence de l'IA - IPR - LCC. Cet examen vaut aussi pour l'enseignement secondaire pour les non spécialistes, ainsi un professeur d'histoire - géographie pourra recevoir une habilitation pour que l'immersion partielle au sein du collège ou lycée classé site bilingue soit possible. 
Cette habilitation donne droit à un mouvement spécifique dans l'ensemble des postes fléchés par l'Académie ${ }^{5}$.

La charte des sites bilingues s'articule autour de quatre priorités « choisies pour obtenir la meilleure réussite des élèves de l'académie de Corse :

- I - Respecter l'ensemble des objectifs de l'école publique pour la construction des savoirs, des compétences méthodologiques et des attitudes, dans les trois cycles d'enseignement par l'application des programmes spécifiques réglementaires.

- II - Développer l'enseignement de la langue corse « langue de communication et langue de culture » en tant que discipline spécifique mais aussi en usage comme vecteur de la didactique des matières non linguistiques à côté de la langue française.

- III - Adapter les évolutions de la pédagogie, de la connaissance et des outils de la communication moderne, aux richesses du substrat culturel de la Corse.

- IV - Concevoir les missions des professeurs des écoles par optimisation des compétences spécifiques de chaque enseignant mais aussi en recherchant la force et le dynamisme du travail concerté des équipes éducatives d'école et de réseaux. $»^{6}$

\section{Formation, programmes et outils didactiques}

Dans l'attente de la grande réforme de la mastérisation, la formation initiale en Corse s'est adaptée aux concours. Les répartitions horaires se font selon le tableau ci - dessous :

\begin{tabular}{|l|l|}
\hline $\begin{array}{l}\text { Formations LCC dispensées à } \\
\text { L'IUFM de Corse }\end{array}$ & Quantité horaire donnée \\
\hline $\begin{array}{l}\text { PLC1 (Professeurs lycées collèges, préparation au CAPES de } \\
\text { corse) }\end{array}$ & 363 \\
\hline PLC2-LCC (année de certification) & 100 \\
\hline FCI (Formation commune interdisciplinaire) des PLC & $36(18+18$ optionnelles) \\
\hline PE1 (professeurs des écoles préparant le concours) & 50 \\
\hline PE1 bilingues & 100 \\
\hline PE2 (PE stagiaires, formation professionnelle) & 50 (modules débutants et confirmés) \\
\hline PE2 bilingues & $\begin{array}{l}25 \text { perfectionnement } \\
25 \text { didactique de la langue } \\
30 \text { formation à l'enseignement en langue }\end{array}$ \\
\hline
\end{tabular}

Concernant la formation continue, les formations en langue corse sont intégrées au plan académique du second degré (PAF) et aux plans départementaux du premier degré (PDF).

21 On peut constater une présence régulière de propositions de stages. Par exemple, dans le $\operatorname{PAF}^{7}$ 2008/2009, 10 stages ont été proposés pour un volume d'heures de 144 (les stages variant de 6 à 18 heures). On note par ailleurs que conformément aux prescriptions du PRDF (2007, 36), les thématiques sont fortement orientées vers les compétences permettant l'immersion partielle (par exemple : histoire, SVT ou EPS en langue corse).

Cette tendance vers de meilleurs outils donnés à l'immersion partielle est confirmée par le principal éditeur de documents didactiques : le CRDP de Corse ${ }^{8}$.

Depuis sa création en Corse en 1977, le CRDP a publié 140 documents pédagogiques en langue corse (manuels, recueils, récits, films, CD - Rom, ...). Les dernières publications s'attachent tout particulièrement à l'adaptation en langue corse de manuels généraux traitant les programmes d'enseignement en français (par exemple, le tout récent Storia Giugraffia in 3a correspondant au format exact de l'ouvrage de la collection Martin IVERNEL de chez Hatier, avec ses livrets élèves et professeurs). 
Pour les sciences humaines notamment, le débat qui s'ouvre aujourd'hui concerne justement les contenus de ce type d'ouvrages: faut - il se contenter d'adapter un ensemble de manuels ou d'outils didactiques issus de grands éditeurs nationaux ou faut il compléter les collections par des manuels aux contenus spécifiques qui ont trait à la Corse?

Même si elle n'affecte pas directement les programmes, cette dimension éditoriale locale n'est de toute façon pas absente, tant du côté du CRDP que dans l'ensemble de l'édition privée. Il incombe ainsi aux enseignants d'adapter leur programme. Ils peuvent le faire sur leur propre initiative en jouant sur les thèmes transversaux, dans le cadre du projet d'établissement ou encore en s'appuyant sur un corpus de préconisations disponibles auprès du Rectorat et éditées par le CRDP.

6 Sur ses annonces, le CRDP précise encore que les futurs outils d'apprentissage de la langue corse seront conformes aux préconisations du Cadre européen commun de référence pour les Langues (CECRL) du Conseil de l'Europe. Au niveau régional, les engagements futurs s'inscriront dans le plan de développement de la LCC et du multilinguisme 2007 2013 entre l'État et la CTC.

Ces résultats, cette production d'ouvrages (y compris dans l'édition privée pouvant prétendre à des subventions) sont essentiellement dus aux moyens mis à disposition par les contrats de plan. Ainsi dans la convention État - CTC d'octobre 2003, la programmation des manuels de disciplines devait couvrir d'ici la fin du Contrat de Plan 2000 - 2006 tous les niveaux du premier degré en Mathématiques et tous les niveaux du collège en Histoire - Géographie et en Mathématiques.

L'objectif de bilinguisme demeure un affichage récurrent depuis les années 2000. On peut ainsi lire dans le PRDF $(2007,25)$ : « Sur la durée du Plan (2007 - 2013), partout où cela est possible la généralisation de l'enseignement bilingue commencé à l'école maternelle doit se poursuivre jusqu'à la fin du primaire. $»^{9}$ Il précise par ailleurs dans son bilan d'étape, l'état de la consommation des importants crédits accordés :

\begin{tabular}{|c|c|c|}
\hline \multicolumn{3}{|l|}{ ARTICLE 16} \\
\hline $\begin{array}{l}\text { La CTC poursuit son action d'accompagnement } \\
\text { telle qu'elle a été prévue dans le Contrat de Plan } \\
2000-2006 \text {. }\end{array}$ & $\begin{array}{l}\text { CTC: } 1,9 \mathrm{MI} \epsilon \\
\text { Réalisation } 2000-2004: 80 \% \\
\text { - Aides aux sites bilingues; } \\
\text { - Aides aux Centres de séjours; } \\
\text { - Edition; } \\
\text { - Diffusion; } \\
\text { - Sections bilingues et méditerranéennes } 2^{\text {nd }} \text { degré ; } \\
\text { - Ateliers de langue corse. }\end{array}$ & $\begin{array}{l}\text { ETAI : } 1,9 \mathrm{MI} \epsilon \\
\text { Réalisation } 2000-2004: 73,63 \% \\
\text { - Aides aux sites bilingues } \\
\text { - Aides aux Centres de séjours } \\
\text { - Edition } \\
\text { - Diffusion } \\
\text { - Sections bilingues et méditerranéennes } \\
2^{\text {nd }} \text { degré } \\
\text { - Ateliers de langue corse }\end{array}$ \\
\hline
\end{tabular}

9 Cet extrait nous permet de préciser encore l'existence de Centres de séjours en immersion ; ils sont au nombre de trois, tous situés en Haute - Corse et permettent à des classes de fréquenter la langue corse de façon intensive (même si l'immersion totale est difficile à atteindre), sur des thématiques spécifiques (nature, milieu rural, parcours patrimoine urbain).

30 Ajoutons la quasi - finalisation des programmes officiels disponibles sur Bulletin officiel de l'Education nationale (BOEN) ${ }^{10}$. Tous les niveaux primaire et collège sont accessibles, seule la partie lycée demeure à proposer.

Enfin, on notera pour la formation adulte une innovation importante : la création du CLC (Certificatu di Lingua Corsa). Ce certificat a été élaboré dans le cadre d'une réflexion commune entre les services académiques représentés par les GRETA et l'Université de 
Corse. Conforme aux niveaux du Cadre européen, il est présenté11 comme "une certification de l'Éducation nationale adaptée aux besoins de la société et notamment du monde professionnel. »

\section{Des problèmes et des enjeux nouveaux}

Pour ce qui touche à la normalisation, la langue corse s'est appuyée sur un développement original : la norme polynomique, c'est - à - dire plurielle, qui cultive l'intertolérance dialectale. Cette conception nouvelle de la norme linguistique, portée par les universitaires corses et vulgarisée par le système scolaire à travers des enseignants de corse spécialement formés à l'enseignement polynomique, a été d'autant mieux accueillie par la société civile qu'elle épousait en quelque sorte une réalité sociolinguistique vivante bien qu'inconsciente puisque relevant des attitudes. La culture d'une certaine conscience métalinguistique libère la parole, car elle ôte à la langue tout pouvoir discriminatoire. Cela a permis une évolution notable des mentalités enlisées au départ dans la gangue diglossique.

Le débat sur la norme semble aujourd'hui réduit à quelques cas qui ne manqueront pas d'être réglés au sein du large consensus obtenu par le double effet d'une codification cohérente proposée en 1971 et d'un appareil critique sociolinguistique basé sur la polynomie et le plurilinguisme dans la société. Cette dernière option est d'ailleurs largement affichée par la CTC qui, dans un consensus politique très fort, la modélise ainsi (PRDF 2007, 50) :

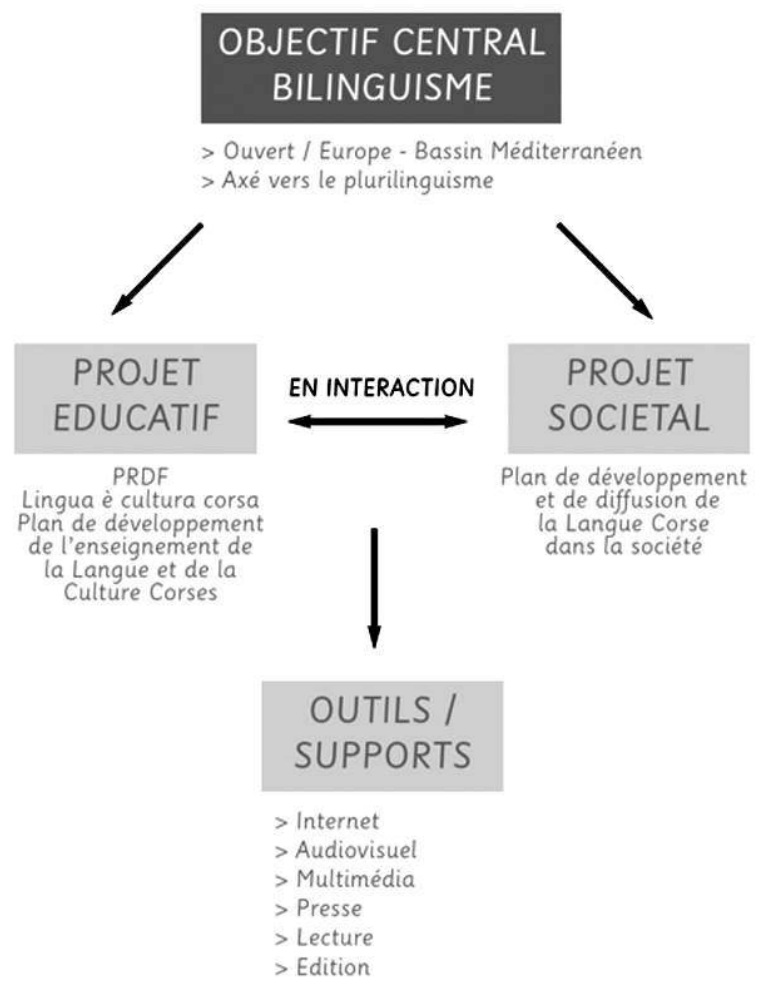

Pourtant l'enseignement du corse connaît encore des problèmes importants, malgré les incontestables progrès liés aux différents acteurs militants, associatifs et institutionnels. Il s'agit tout d'abord de traduire en actions cet affichage à partir d'une évaluation qui dépasse la simple statistique basée sur le quantitatif (effectifs, maîtres, moyens) et sur le 
déclaratif comme méthode la plus fréquente des enquêtes. Comment connaitre plus finement la quantité réelle de l'enseignement dispensé, par exemple?

Il est urgent aujourd'hui d'aller vers des diagnostics plus précis sur la qualité de l'enseignement. Car le véritable problème se situe justement dans cette massification très bien attestée par l'ensemble des enquêtes mais que l'on connaît finalement assez mal.

Si on a pu identifier depuis une vingtaine d'années un réseau de militants à l'expérience affirmée ${ }^{12}$, on a du mal aujourd'hui à évaluer l'état réel du chantier pédagogique : quelle est la pratique de ces jeunes maîtres issus du concours? La langue corse peut - elle servir de cheval de Troie pour une réussite moins concurrencée aux concours de recrutement? Comment mesurer la véritable motivation des nouveaux habilités qui visent parfois le statut de maître bilingue afin de se donner une mobilité plus sûre? La mise en place de cursus différents (systèmes standard / bilingue) a - $\mathrm{t}$ - elle des effets cachés ? Y a - $\mathrm{t}$ - il une obligation à honorer son statut bilingue de façon pérenne? Parmi toutes les questions, la plus lancinante s'adresse directement à l'efficacité du système : pourquoi, avec de tels moyens, l'école ne produit - elle pas de corsophones?

La réponse est sans doute complexe et appelle une analyse systémique qui affranchirait pour partie la responsabilité de l'école. Mais il est clair qu'un constat trop optimiste ne saurait être de mise et que l'affichage, ô combien louable, présenté plus haut doit se donner les moyens d'être atteint car l'hypothèse d'une hypocrisie politique n'est pas totalement levée.

En 2007, l'Assemblée de Corse a voté à l'unanimité un plan de développement de la langue corse pour la période 2008 - 2013. Le dernier paragraphe dit: "La valorisation et le développement du plurilinguisme constituent ainsi l'un des aspects fondamentaux des politiques d'inclusion sociale et d'éducation à une citoyenneté démocratique, qui ne peut être qu'interculturelle » (Rapport Lingua corsa : un fiatu novu in Thiers, 2008, 325).

Cette dernière assertion pose bien tout l'enjeu sociétal. Il ne concerne pas seulement la sauvegarde d'une langue régionale mais pose ou propose un modèle de société où le commun ne prétend plus à l'exclusivité linguistique et où le monolinguisme est un modèle caduc. Malgré des progrès évidents, la Corse est à ce titre à la croisée des chemins entre l'innovation nécessaire et les tentations conservatistes ou l'idéologie diglossique non encore totalement dépassées. À notre avis, l'État ne pourra, dans le cadre d'une prochaine loi, aller beaucoup plus loin que ce que la Corse a déjà promu. L'étape nationale, dans une volonté de construction de cet espace d'avenir qu'est l'Europe, demeure fortement liée à la ratification de la Charte européenne des langues minoritaires. Ira - $\mathrm{t}$ - on vers ce type de reconnaissance pour les langues de France?

$\mathrm{Du}$ coté corse, on constate que sauver une langue régionale est un chantier ardu, avide de moyens et malgré tout incertain. Mais il est clair que le sens donné aux choix politiques relève d'une quête de nouveaux modèles du vivre ensemble dans des espaces devenus complexes par l'histoire et la mobilité des groupes humains à respecter.

\section{BIBLIOGRAPHIE}

« Rapport final présenté au Cunsigliu di a lingua è di a cultura corsa, Lingua corsa : un fiatu novu » (janvier 2007) in THIERS, J. (2008) Papier d'identité(s). Ajaccio : Albiana ou «Plan

stratégique d'aménagement et de développement linguistique pour la langue corse 2007 - 2013 » 
adopté à l'unanimité par l'Assemblée de Corse le 26 juillet 2007 : http://www.corse.fr/education/ corse $/$ ?id $=5 \& \mathrm{id} 2=24$

QUENOT, S. (2009). La langue corse. Bastia : Anima corsa.

PRDF. Plan Régional de Développement de la Formation. (2007). Livret 6, La langue et culture corse http://www.corse.fr/documents/education/PRDF/livret \%206 \%20web.pdf (consulté le 10/04/2009).

IGEN (2006) Évaluation de l'enseignement dans l'académie de corse, Rapport - n²006 - 39 de juillet 2006 de l'Inspection Générale de l'Éducation Nationale,

http://media.education.gouv.fr/file/03/7/3037.pdf (consulté le 10/04/2009).

\section{NOTES}

1. C'est, en effet, en 1974 que sera étendue la loi DEIXONNE à la langue corse lui permettant ainsi d'accéder à un premier statut de langue enseignée dans les établissements publics.

2. La part du privé en Corse représentait, à la rentrée $2008,6,5 \%$ des effectifs élèves. Ce taux est assez stable depuis une trentaine d'années dans une région qui n'a pas une grande tradition d'enseignement privé comme d'autres régions à forte identité (Bretagne ou Pays basque, par exemple).

3. On retiendra essentiellement la circulaire SAVARY de 1982 portant les possibilités d'enseignement à 3 heures semaine, la création des CAPES langues régionales dont le CAPES de langue corse en 1990 (dont la spécificité est d'être monovalent contrairement aux autres bivalents), la circulaire BAYROU de 1995 ayant permis l'ouverture des premiers sites bilingues en Corse, les mesures LANG de septembre 2001 créant les concours de recrutement spécifiques (renforçant par effet induit la formation initiale en IUFM et la formation continue en ouvrant la possibilité d'une habilitation à l'enseignement bilingue pour les maîtres déjà recrutés) et tout dernièrement, l'inscription en juillet 2008 des langues régionales dans la Constitution française à titre de patrimoine.

4. Il s'agit pour la Corse de M. Jean - Marie ARRIGHI, Inspecteur pédagogique régional pour la LCC.

5. On peut trouver la carte des sites bilingues sur le site de l'académie de Corse http ://www.accorse.fr/lcc_20/E-scole-bislingue-2005_a2.html

6. Le texte de cette charte est disponible sur le site de l'académie de Corse http://www.accorse.fr/lcc_20/Cartula-di-e-scole-Bislingue_a32.html (consulté le 10/04/2009).

7. Plan académique de Formation

8. CRDP, Centre Régional de Documentation Pédagogique. On peut trouver un ensemble d'informations sur l'édition concernant la langue corse sur http://www.crdp-corse.fr/index.php/ lcc (consulté le 10/04/2009).

9. Extrait de la délibération $n^{\circ} 05 / 112$ de l'Assemblée de Corse, approuvant les orientations stratégiques pour le développement et la diffusion de la langue corse (annexes p. 26). http:// www.corse.fr/education/corse/?id=5\&id2=24 (consulté le 10/04/2009).

10. On retrouve ces programmes en téléchargement sur le site de l'Académie de Corse : http:// www.ac-corse.fr/lcc_20/downloads/Testi-e-prugrammi_t79.html (consulté le 10/04/2009).

11. http://www.certificatu.fr/accueil.htm (consulté le 10/04/2009).

12. On retrouve nombre de ces enseignants du public regroupés au sein de l'association «A scioglilingua » ayant son siège à Corte 


\section{RÉSUMÉS}

La langue corse dans l'enseignement a connu durant ces trente dernières années des progrès importants. Malgré un recul de son usage social, sa présence dans l'enseignement bénéficie d'une politique très volontaire de la part de l'État, de la Région et de l'Université. Les années 2000 marquent une direction très nette vers le bilinguisme scolaire posant ainsi l'enjeu du choix d'une société plurilingue.

Corsican language in education through the last thirty years has largly improved. Despite a decline of its social use its presence in education benefits from a very voluntary politics of the State, the region and the University. 2000s underline a very clear direction towards the school bilingualism thus putting the stake in the choice of a multilingual society.

\section{INDEX}

Mots-clés : bilinguisme scolaire, enjeu, progrès

Keywords : progress, school bilingualism, stake

\section{AUTEUR}

\section{ALAIN DI-MEGLIO}

Maître de conférences à l'IUFM de Corse 